\title{
The anti-asthmatic drug, montelukast, modifies the neurogenic potential in the young healthy and irradiated brain
}

Yohanna Eriksson ${ }^{1}$, Martina Boström ${ }^{1,2}$, Åsa Sandelius ${ }^{3}$, Kaj Blennow ${ }^{3,4}$, Henrik Zetterberg ${ }^{3,4,5,6}$, Georg Kuhn ${ }^{7,8}$ and Marie Kalm (1) ${ }^{1}$

\begin{abstract}
Brain tumors are the most common form of solid tumors in children. Due to the increasing number of survivors, it is of importance to prevent long-term treatment-induced side effects. Montelukast, a leukotriene receptor antagonist, may have the desired neuroprotective properties. The aim of the study was to determine whether montelukast could reduce adverse effects of cranial irradiation (CIR) to the young brain. Daily injections of montelukast or vehicle was given to young mice for 4 or 14 days in combination with CIR or under normal conditions. Montelukast treatment for 4 days protected against cell death with $90 \%$ more cell death in the vehicle group compared to the montelukast group $24 \mathrm{~h}$ after CIR. It also resulted in less microglia activation $6 \mathrm{~h}$ after CIR, where montelukast lowered the levels of CD68 compared to the vehicle groups. Interestingly, the animals that received montelukast for 14 days had 50\% less proliferating cells in the hippocampus irrespective of receiving CIR or not. Further, the total number of neurons in the granule cell layer was altered during the sub-acute phase. The number of neurons was decreased by montelukast treatment in control animals (15\%), but the opposite was seen after CIR, where montelukast treatment increased the number of neurons (15\%). The results show beneficial effects by montelukast treatment after CIR in some investigated parameters during both the acute phase and with longer drug treatment. However, it also resulted in lower proliferation in the hippocampus under normal conditions, indicating that the effects of montelukast can be either beneficial or unfavorable, depending on the circumstances.
\end{abstract}

\section{Introduction}

Children with asthma receive daily treatment and according to international guidelines the first choice of treatment is inhaled corticosteroids. The treatment is often combined with adrenergic $\beta_{2}$ receptor agonists. The second choice for treatment is leukotriene receptor antagonists, for example, montelukast (Singulair $\left.{ }^{\circledR}\right)$, which is approved for use in children $(<12 \text { years })^{1}$. It is not unusual that the first and second choices

\footnotetext{
Correspondence: Marie Kalm (marie.kalm@gu.se)

${ }^{1}$ Department of Pharmacology, Institute of Neuroscience and Physiology,

Sahlgrenska Academy, University of Gothenburg, Gothenburg, Sweden

${ }^{2}$ Department of Oncology, Institute of Clinical Sciences, Sahlgrenska Academy,

University of Gothenburg, Gothenburg, Sweden

Full list of author information is available at the end of the article.

Edited by B. Joseph
}

are combined. Montelukast has been considered well tolerated in children due to mild and transient side effects $^{2}$. However, current reports show that montelukast treatment can cause psychiatric adverse drug reactions in children, such as sleep disorder, anxiety, aggressiveness, and hyperactivity with an incidence higher than $10 \%^{3-5}$. The underlying reasons for these side effects remain unknown.

Leukotrienes are normally present at low levels in the brain but increase during pathological conditions ${ }^{6}$. Montelukast has been described to be neuroprotective, for example, in the aging brain and after focal cerebral ischemia $^{7,8}$. In the aging brain, increased activation of microglia is accompanied with decreased hippocampal neurogenesis and cognitive decline ${ }^{7}$. Daily, oral

\section{(c) The Author(s) 2018}

(c) (i) Open Access This article is licensed under a Creative Commons Attribution 4.0 International License, which permits use, sharing, adaptation, distribution and reproduction cc) in any medium or format, as long as you give appropriate credit to the original author(s) and the source, provide a link to the Creative Commons license, and indicate if changes were made. The images or other third party material in this article are included in the article's Creative Commons license, unless indicated otherwise in a credit line to the material. If material is not included in the article's Creative Commons license and your intended use is not permitted by statutory regulation or exceeds the permitted use, you will need to obtain permission directly from the copyright holder. To view a copy of this license, visit http://creativecommons.org/licenses/by/4.0/. 
administration of montelukast to rats for 6 weeks improved these negative changes in the aging brain ${ }^{7}$, effects that could also be beneficial in radiotherapyinduced injury in children.

Brain tumors are the most common form of solid tumors in children ${ }^{9}$, with surgery, chemotherapy, and radiotherapy as the main treatment strategies ${ }^{10}$. All of these treatments cause late side effects, with radiotherapy having the highest severity when graded ${ }^{11}$. Side effects range from sleep disturbance to cognitive impairment ${ }^{12}$. The mechanisms of chronic radiation-induced damage involve, for example, long-term toxicity to neural cell types, including stem and progenitor cells, loss of oligodendrocytes, and inflammatory responses ${ }^{13}$. Cranial radiotherapy also changes the chemical milieu and affects supporting cells such as microglia ${ }^{14,15}$. The subgranular zone (SGZ) in the hippocampus, an area in the brain that harbor stem cells, is very sensitive to radiotherapy in both the young and adult brain, and loss of these cells may contribute to cognitive deficits ${ }^{16-18}$. Finding means to ameliorate radiation-induced injury is of great interest for the increasing number of long-term childhood cancer survivors.

Targeting the irradiation-induced inflammatory response is of interest to prevent negative effects on cognition and neurogenesis. Inhibiting microglia with MW-151, a selective inhibitor of proinflammatory microglial cytokine production, restored hippocampal-dependent learning, improved synaptic function, and partially protected neurogenesis after cranial irradiation (CIR) to the adult rat brain $^{19}$. Further, it has been shown that indomethacin, a common nonsteroidal anti-inflammatory drug, has the potential to partly increase neurogenesis after CIR in adults ${ }^{20}$. Blocking chemokine $(\mathrm{C}-\mathrm{C}$ motif) receptor 2 (Ccr2) has also prevented neuronal dysfunction and hippocampal-dependent memory dysfunction induced by irradiation toward the adult mouse brain ${ }^{21}$. We have previously shown that the juvenile and adult brain have different radiation-induced inflammatory responses ${ }^{22}$, which is of importance if using an anti-inflammatory approach to protect the brain from CIR-induced injury. In the developing brain, it has been shown that blocking the complement cascade can improve reversal learning after CIR, but without effects on neurogenesis ${ }^{23}$. As mentioned earlier, montelukast is a leukotriene receptor antagonist. Leukotrienes are lipid mediators of inflammation and are metabolized from arachidonic acid through the 5-lipoxygenase (5-LOX) pathway. It has been shown that minocycline, a tetracycline antibiotic that blocks the activation of 5-LOX, had positive effects on cognitive impairment and decreased apoptosis in newborn neurons $\left(\mathrm{DCX}^{+}\right)$following a single dose of $20 \mathrm{~Gy}$ irradiation to the brain of 1 month old rats ${ }^{24}$. Inhibiting the 5-LOX pathway to target the inflammatory response could therefore be an interesting strategy to investigate when trying to protect the normal tissue during radiotherapy. The purpose of this study was to investigate the effect of montelukast in combination with CIR to the young brain.

\section{Results}

The study outline is presented in Fig. 1a. Weight gain was carefully monitored for all mice injected daily for 14 days and an interaction between treatment, drug, and time was observed ( $P=0.036$, Fig. $1 b-c)$. CIR resulted in a significantly delayed weight gain compared to control animals and stayed below the other groups throughout the experiment. Interestingly, the montelukast group did not show the same delayed weight gain following CIR.

\section{Acute phase following CIR}

To investigate the acute CIR-induced injury, caspase activity in brain homogenate and neurofilament light chain (NFL) in serum was measured. Cell death (caspase$3 / 7$-activity) increased to similar levels in both the vehicle and the montelukast group at $6 \mathrm{~h}$ after CIR $(P=0.0004$, Fig. 2a). The relative increase after CIR was $125 \%$ in the vehicle groups (post hoc, $P=0.0121$ ) and $141 \%$ in the montelukast groups (post hoc, $P=0.0116$ ). Interestingly, the levels of caspase activity had decreased back to normal levels at $24 \mathrm{~h}$ after CIR. However, there was a drug effect at this time $(P=0.0078$, Fig. 2 a) with $90 \%$ more cell death in the CIR vehicle group compared to the CIR montelukast group (post hoc, $P=0.0357$ ). The level of NFL, reflecting neuronal injury, increased in serum $6 \mathrm{~h}$ after CIR $(P=0.0055$, Fig. $2 \mathrm{~b})$. This trend was seen in both the vehicle (post hoc, $58 \%, P=0.1136$ ) and the montelukast groups (post hoc, 77\%, $P=0.0565$ ). However, no difference was observed between the different groups at $24 \mathrm{~h}$ after CIR.

Cluster of differentiation 68 (CD68) and chemokine (C-C motif) ligand 2 (CCL2) were measured in brain homogenate to evaluate the inflammatory response following CIR. The level of CD68 was significantly altered $6 \mathrm{~h}$ after CIR $(P=0.0128$, Fig. 2c). At this time point montelukast lowered the levels of CD68 compared to both vehicle groups (post hoc, n.s.). The levels of CCL2 increased significantly after $\mathrm{CIR}$ in both vehicle and montelukast groups at both time points $(P<0.0001$, Fig. 2d). The vehicle group showed a CIR-induced increase of $298 \%$ (post hoc, $P<0.0001$ ) and the montelukast group a CIR-induced increase of $254 \%$ (post hoc, $P<0.0001) 6 \mathrm{~h}$ after the injury.

\section{Sub-acute phase following CIR}

Twelve days after CIR, the volumes of corpus callosum, thalamus, and dentate gyrus were measured (Fig. 3a-b). The volume of corpus callosum was smaller following CIR for both vehicle and montelukast groups $(P=0.0115$, 


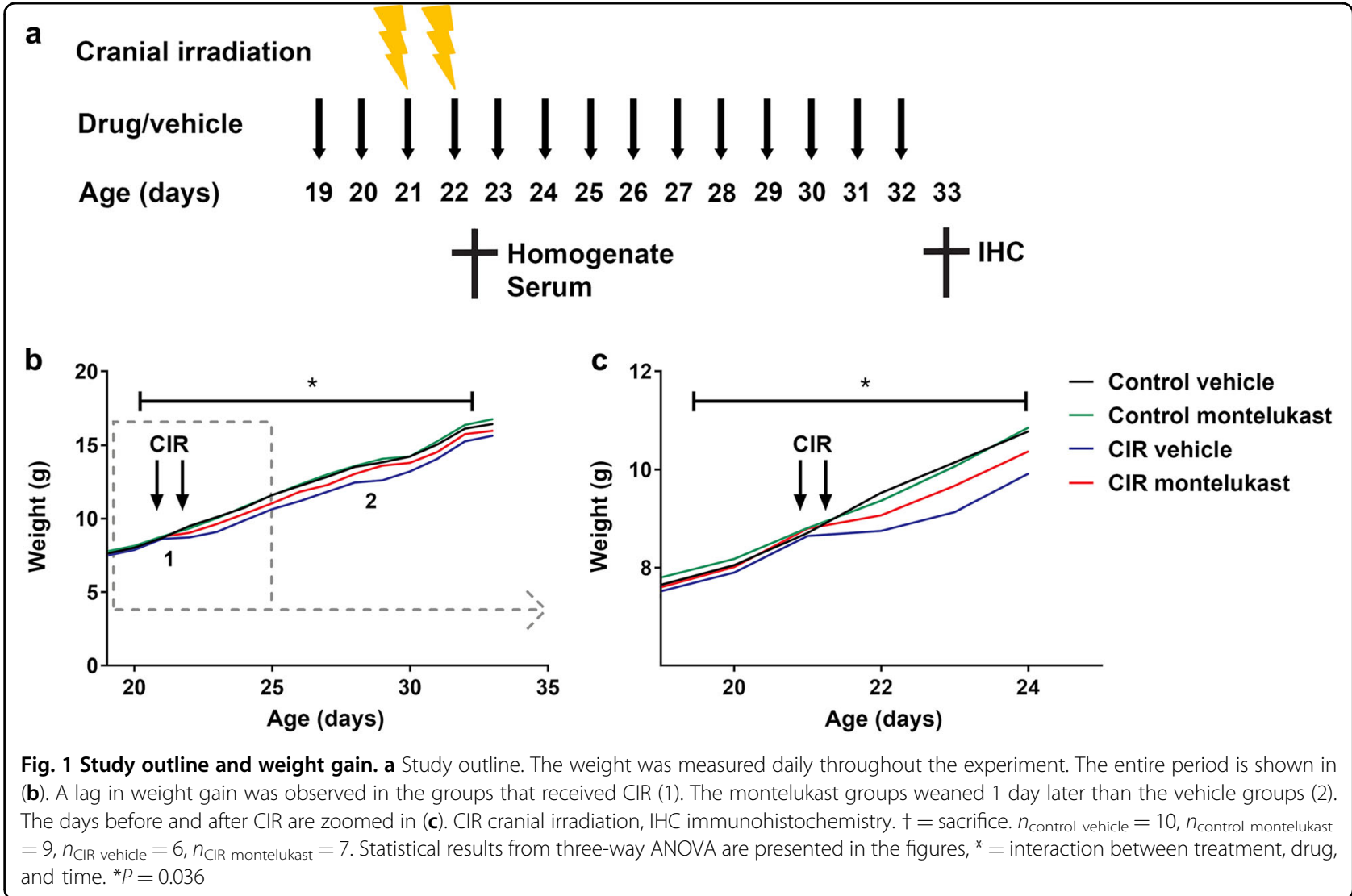

Fig. 3c). For vehicle animals, it was $25 \%$ smaller (post hoc, $P=0.0334$ ) and for the montelukast group the decrease was $13.4 \%$ (post hoc, n.s). No difference was observed in the thalamic region or the dentate gyrus (Fig. 3d-e). However, an analysis of the subregions in the dentate gyrus revealed that the volumes of the granule cell layer (GCL, $P=0.0228$, Fig. $3 f)$ and hilus $(P=0.0243$, Fig. 3 h) changed following CIR. The CIR vehicle group had a smaller volume compared to the control vehicle group in the GCL (9.8\%, post hoc, n.s.) and the hilus (16.6\%, post hoc, n.s.). The CIR montelukast group also had a smaller volume compared to the non-irradiated montelukast group in the GCL $(17.4 \%$, post hoc, n.s., $P=0.0827)$ and the hilus (16.0\%, post hoc, n.s.). There was no difference in the molecular layer (ML) (Fig. 3h).

Cellular effects in the GCL were assessed by determining the total numbers of neurons (Neurotrace ${ }^{\mathrm{TM}}$ ), proliferating cells $\left(\mathrm{Ki}-67^{+}\right)$and newborn neurons (doublecortin, $\mathrm{DCX}^{+}$). Analysis of the total number of neurons revealed a significant interaction between treatment and drug $(P=0.0364$, Fig. $4 \mathrm{a}-\mathrm{b})$. The vehicle CIR group had $23 \%$ less neurons in the GCL compared to vehicle controls. This was not observed in the montelukast group, where CIR did not alter the levels of neurons. Interestingly, montelukast treatment decreased the number of neurons with $15 \%$ when comparing vehicle controls and montelukast controls. Proliferation was assessed by quantifying the number of Ki-67 $7^{+}$cells (Fig. 4c-d). Montelukast decreased proliferation during normal conditions with $50 \%$ (post hoc, $P=0.0291$ ), while CIR did not significantly alter the proliferation levels. The total number of newborn neurons $\left(\mathrm{DCX}^{+}\right)$was decreased in the GCL after CIR but not altered by montelukast $(P<0.0001$, Fig. $4 \mathrm{e}-\mathrm{f})$. CIR treatment decreased the level of newborn neurons with $63 \%$ in the vehicle CIR group compared to vehicle controls (post hoc, $P<0.0001)$ and $55 \%$ in the montelukast CIR group compared to montelukast controls (post hoc, $P<0.0001$ ).

To investigate effects on non-neuronal cells in the dentate gyrus, microglia $\left(\mathrm{Iba}^{+}\right)$, oligodendrocytes $\left(\mathrm{Olig} 2^{+}\right)$, and astrocytes $\left(\mathrm{S} 100^{+}\right.$, possibly including a subpopulation of neurons) were quantified. The number of microglia was affected by both montelukast treatment $(P=0.0233)$ and CIR $(P=0.015$, Fig. 5a-b). However, the post hoc test only revealed a difference between the vehicle control group and the montelukast CIR group in GCL (post hoc, $P=0.0066$ ). Similar trends were observed in the hilus and ML. Further, the number of oligodendrocytes decreased following CIR in GCL $(P<0.0001)$, hilus $(P=0.00005)$, and ML ( $P=0.000243$, Fig. $5 \mathrm{c}-\mathrm{d})$. No effect was observed following montelukast treatment. In the GCL, the vehicle CIR group had 23\% less 


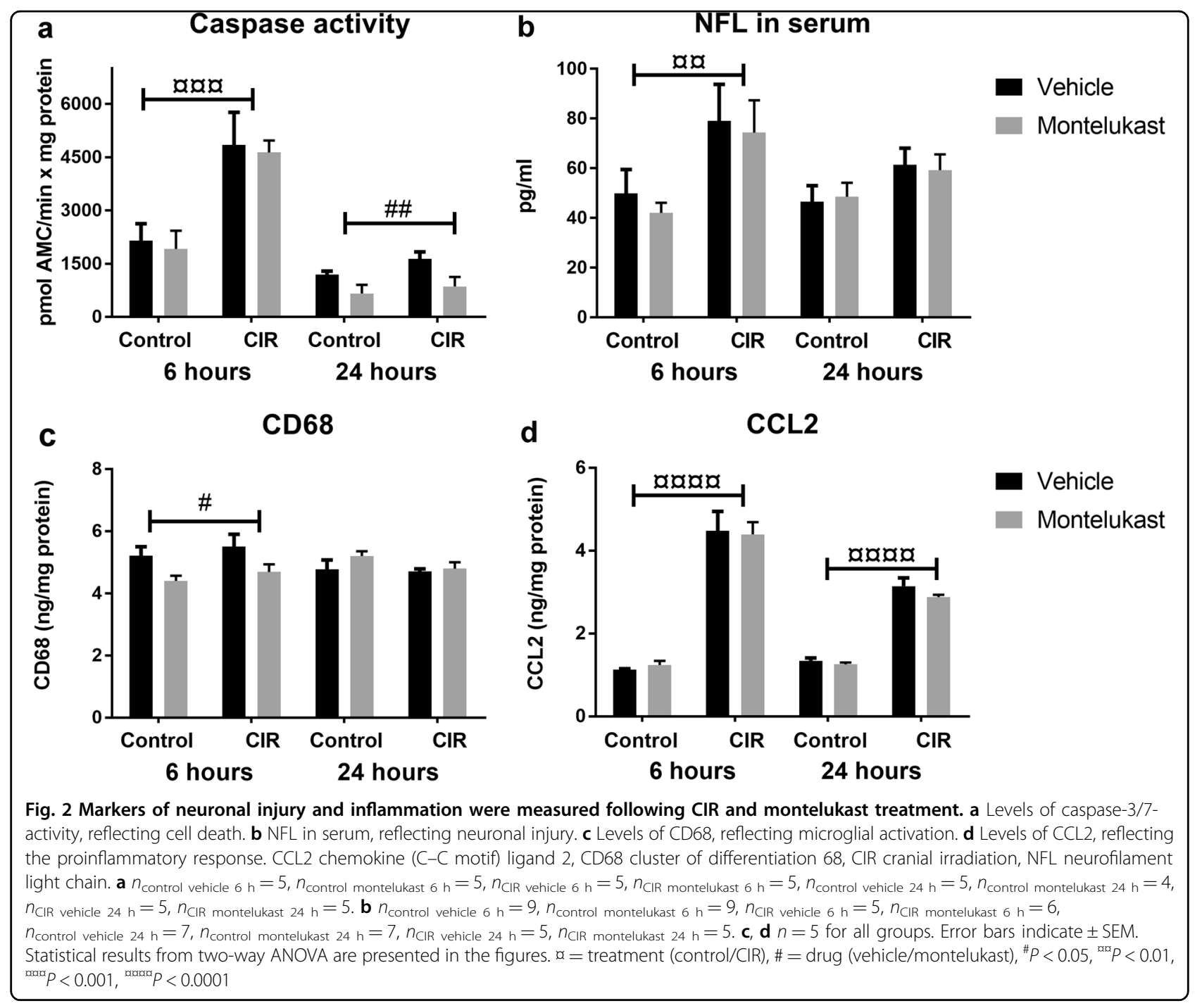

oligodendrocytes compared to the vehicle controls (post hoc, $P=0.039$ ). For the montelukast CIR group, the relative reduction of oligodendrocytes in the GCL was $38 \%$ compared to the montelukast control group (post hoc, $P=0.0007)$. Similar changes were observed in the hilus and the ML for both vehicle and montelukast groups. The number of $\mathrm{S} 100^{+}$cells was not affected by montelukast or CIR treatment (Fig. $5 \mathrm{e}-\mathrm{f}$ ).

\section{Discussion}

Radiotherapy is an effective treatment for brain tumors but unfortunately causes long-lasting side effects in the brain. In this study, parameters that are known to be affected by CIR were investigated to evaluate the effect of montelukast on radiation-induced injury in the developing brain. The major findings were the following: (1) Montelukast treatment resulted in reduced cell death during the acute phase after CIR. (2) The number of neurons was altered by montelukast treatment with a positive effect after CIR, but with a negative effect during normal conditions. (3) Proliferation in the hippocampal neurogenic zone decreased by montelukast treatment. These data indicate that the effects of montelukast can be either beneficial or unfavorable, depending on the physiological conditions.

Delayed weight gain has previously been observed after CIR to the developing brain ${ }^{25}$, but the underlying mechanism is not clear. Two possibilities, which are often seen in patients, are alterations in the hormonal balance or injury to the mucous membrane. A CIR-induced injury in the mucosa in the mouth and throat would make it painful for the mice to eat. Regarding the hormonal levels, the thyroid hormones FT3 and FT4 have been shown to be unaffected 4 months after CIR in a similar mouse model ${ }^{26}$. Presumably, the delayed weight gain is an effect of both hypothalamic-pituitary axis dysfunction 

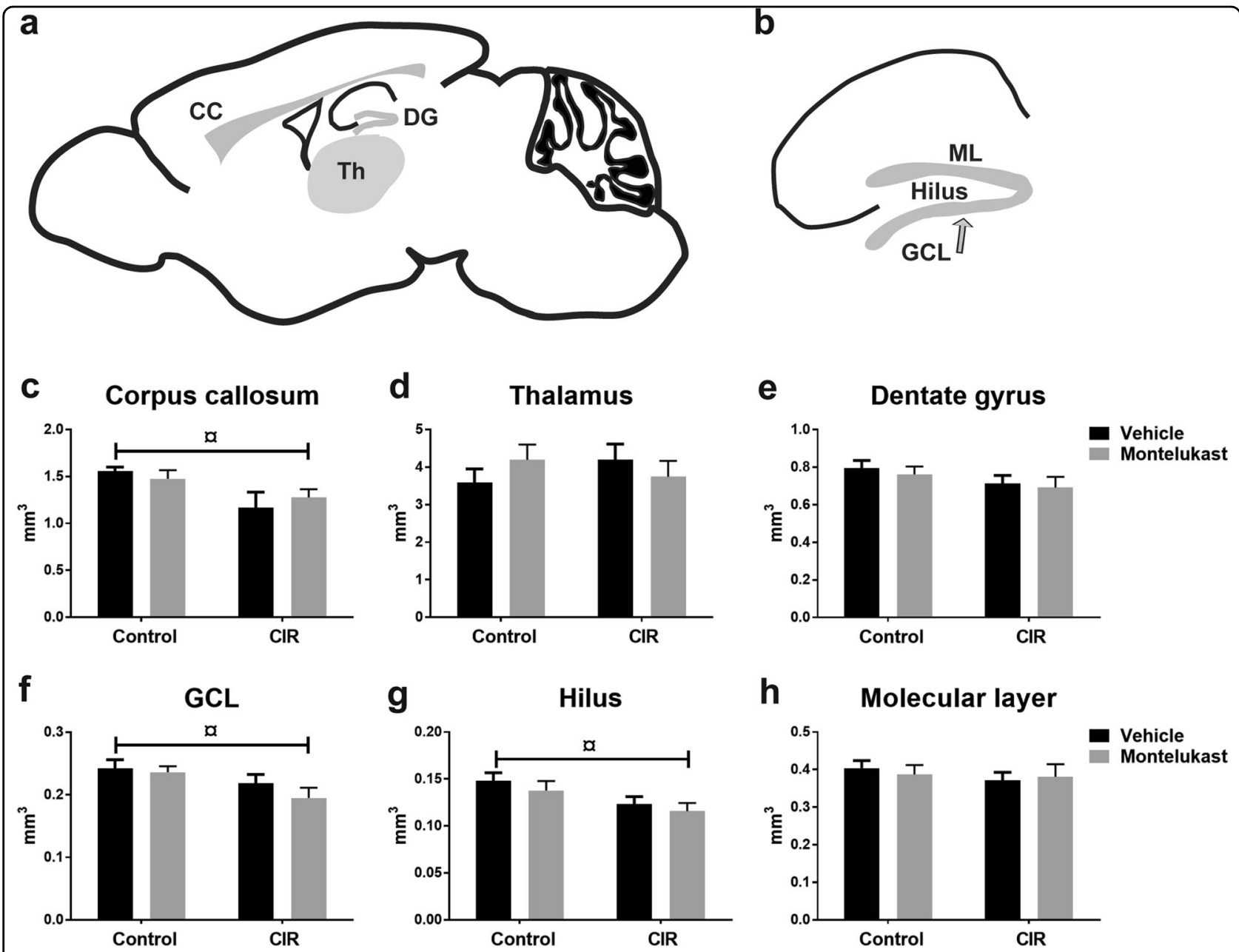

Fig. 3 Volumetric alterations following CIR and montelukast treatment. a Investigated areas are highlighted in the schematic overview. The hippocampal regions are illustrated in $\mathbf{b}$. The volumes were measured in the corpus callosum (c), thalamus (d), and hippocampal dentate gyrus (e). The subregions of the dentate gyrus; GCL (f), hilus $(\mathbf{g})$, and molecular layer $(\mathbf{h})$ are also presented. CC corpus callosum, CIR cranial irradiation, DG dentate gyrus, GCL granule cell layer, $\mathrm{ML}$ molecular layer, Th thalamus. $\mathbf{c} n_{\text {control vehicle }}=7, n_{\text {control montelukast }}=9, n_{\text {CIR }}$ vehicle $=6$, $n_{\text {CIR montelukast }}=5 . \mathbf{d} n_{\text {control vehicle }}=9, n_{\text {control montelukast }}=9, n_{\text {CIR vehicle }}=6, n_{\text {CIR montelukast }}=7, \mathbf{e}, \mathbf{f}, \mathbf{g}, \mathbf{h} n_{\text {control vehicle }}=8, n_{\text {control montelukast }}=9$, $n_{\mathrm{CIR}}$ vehicle $=6, n_{\mathrm{CIR}}$ montelukast $=5$. Error bars indicate $\pm S E M$. Statistical results from two-way ANOVA are presented in the figures, $\boldsymbol{x}=$ treatment (control/CIR), ${ }^{\text {a }} P<0.05$

and injury in the mucous membrane. Here, CIR caused a significantly delayed weight gain in the vehicle group but not in the montelukast group. The different reactions after CIR suggest that montelukast may have a protective effect on the physiological response to CIR, possibly due to its anti-inflammatory effects.

Acute cell death and increased levels of the neuronal injury marker, NFL, in serum are two expected findings following CIR, especially in a still developing brain ${ }^{27,28}$. In this study, the vehicle group exhibited more cell death compared to the montelukast group acutely following CIR. It has been demonstrated that montelukast could increase the proliferation of neuronal precursor cells in vitro through the receptors CysLT1R and GPR $17^{29}$. However, there was no effect on cell fate or differentiation in that study. In this in vivo study, we observed the opposite. Montelukast rather had a negative effect on both proliferation and maturation under control conditions. It is possible that montelukast administration immediately negatively affected the cell proliferation, resulting in less proliferating cells at the time of CIR. This would then be reflected by less cell death. Further, it has been shown that microglia express, for example, GPR $17^{30,31}$, and it is therefore possible that montelukast have a direct impact on microglia which would affect both the levels of CD68 and Iba1. Also, microglia themselves could affect the injury since they are known producers of reactive oxygen species and proinflammatory factors ${ }^{32}$, and excess of these factors can worsen brain injury. Interestingly, montelukast had a positive effect on the 
a

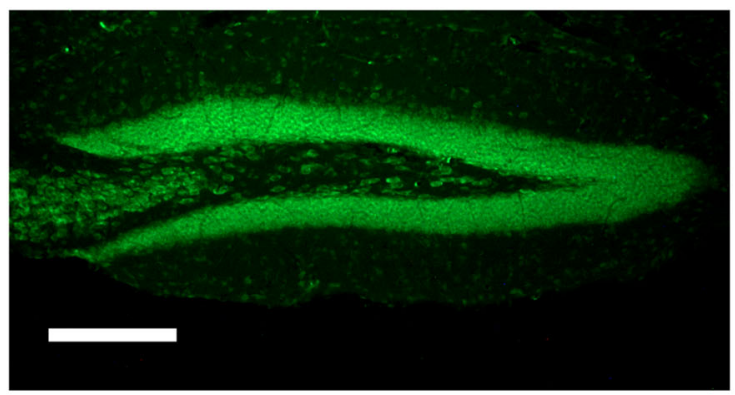

C

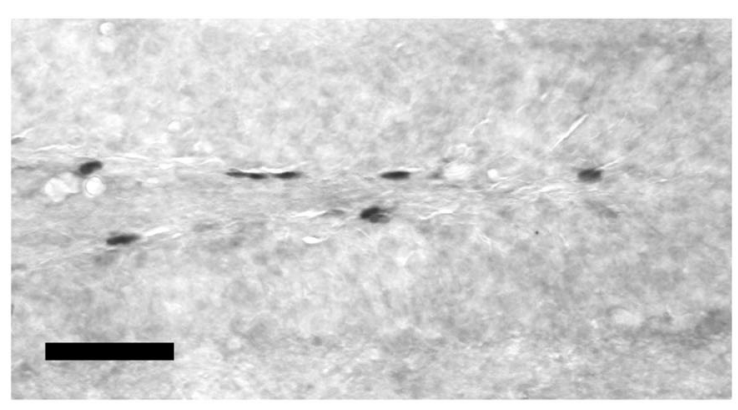

e

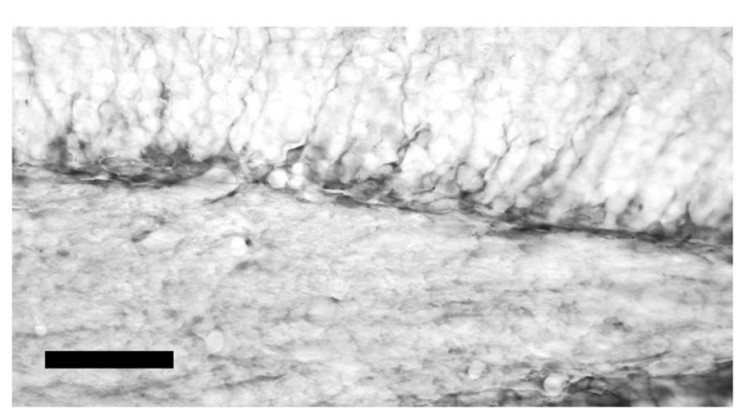

b

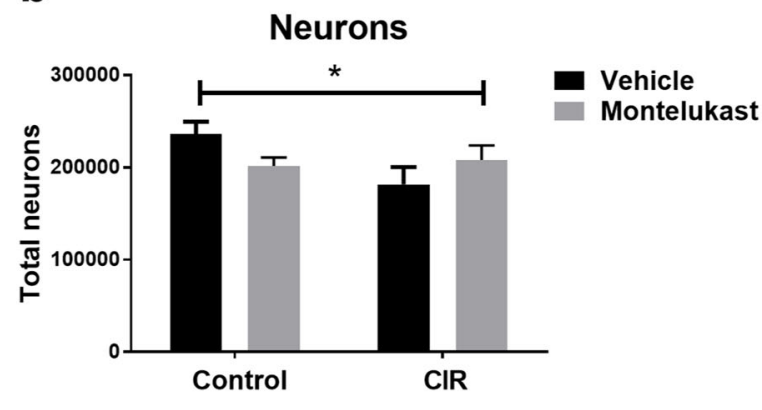

d

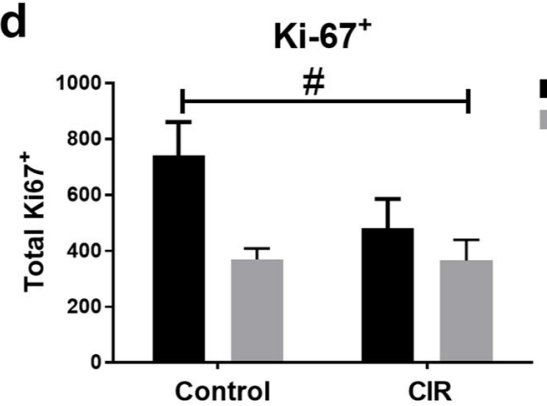

f

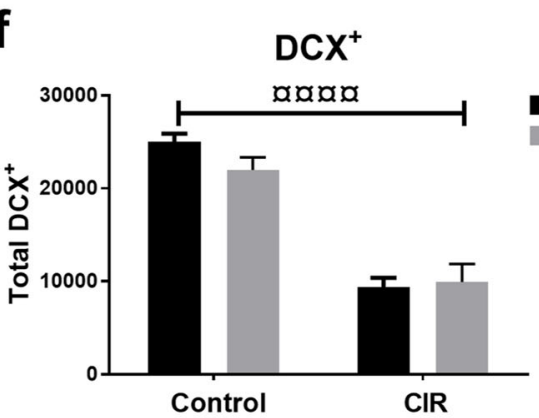

Vehicle

Montelukast

Vehicle

Montelukast

Fig. 4 Cellular effects in the GCL following CIR and montelukast treatment. a A microphotograph of Neurotrace ${ }^{T M}$ in the dentate gyrus. b Neurons were quantified in the GCL where a significant interaction was observed. c Proliferating cells, assessed by Ki-67 ${ }^{+}{\text {staining. } \mathbf{d} \text { Ki- } 67^{+}}^{+}$ quantification in the hippocampal neurogenic SGZ. e Newborn cells, measured as total numbers of DCX+ cells. $\mathbf{f}$ DCX ${ }^{+}$quantification in the SGZ. a Scale bar $20 \mu \mathrm{m}$. $\mathbf{b} n_{\text {control vehicle }}=8, n_{\text {control montelukast }}=9, n_{\text {CIR vehicle }}=6, n_{\text {CIR montelukast }}=6 . \mathbf{c}$ Scale bar $50 \mu \mathrm{m}$. $\mathbf{d} n_{\text {control vehicle }}=9, n_{\text {control montelukast }}$ $=8, n_{\mathrm{CIR}}$ vehicle $=5, n_{\mathrm{CIR} \text { montelukast }}=4$. e Scale bar $50 \mu \mathrm{m}$. $\mathbf{f} n_{\text {control vehicle }}=8, n_{\text {control montelukast }}=9, n_{\mathrm{CIR}}$ vehicle $=6, n_{\mathrm{CIR}}$ montelukast $=5$. CIR cranial irradiation, DCX doublecortin, GCL granule cell layer, SGZ subgranular zone. Error bars indicate \pm SEM. Statistical results from two-way ANOVA are presented in the figures, $a=$ treatment (control/CIR), \# = drug (vehicle/montelukast), ${ }^{*}=$ interaction between treatment and drug. ${ }^{*} P<0.05$, ${ }^{\#} P<0.05,{ }^{\text {axax }} P<0.0001$

number of mature neurons after CIR, but not on the number of proliferating cells or newborn neurons. Hence, the survival of newborn neurons was positively affected in this scenario.

Montelukast has been tested in neurological conditions where inflammation and cognitive dysfunction is of interest. For example, montelukast acutely protects cerebral tissue in neonatal rats following ischemic brain damage $^{33}$. These findings are in line with our results with lower levels of cell death in the montelukast group following CIR. Another study has shown beneficial effects on spatial memory and cognition in the aging brain after montelukast treatment ${ }^{7}$. An in vitro study showed an increased proliferation of neuronal progenitors after montelukast administration, but using higher doses it decreased proliferation ${ }^{29}$. This support our finding that montelukast could have a negative effect on neuronal proliferation in the intact juvenile brain.

Whether montelukast is beneficial or not seems to depend on the absence or presence of injury in the young brain. Positive results from montelukast treatment have been seen in the aging brains of rats after a dose of $10 \mathrm{mg} / \mathrm{kg}$ body weight ${ }^{7}$. The dose to treat asthma in children (2-5 years old) is however $4 \mathrm{mg}$ /day regardless of body weight. In this study, we have examined the effects of montelukast in the young brain after CIR at 
a

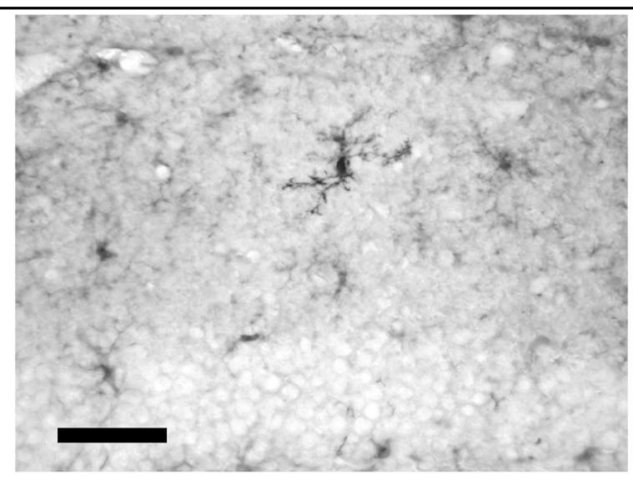

C

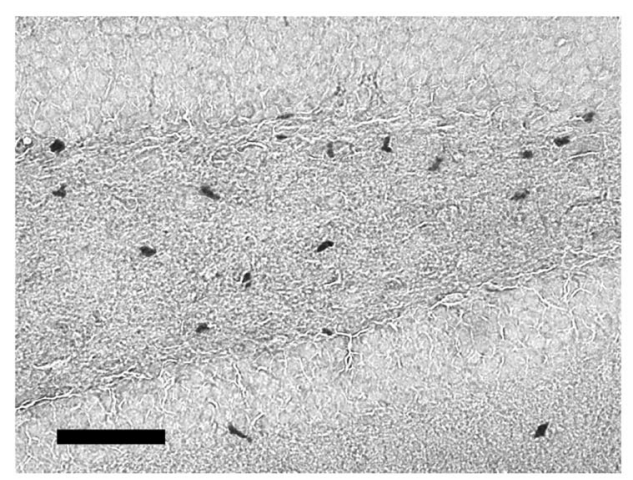

e

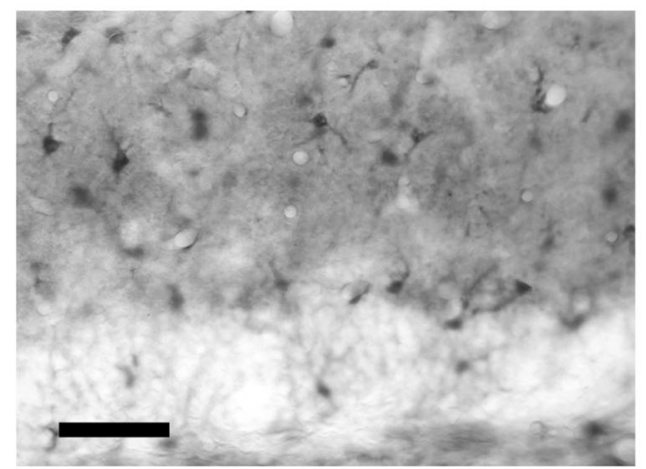

b

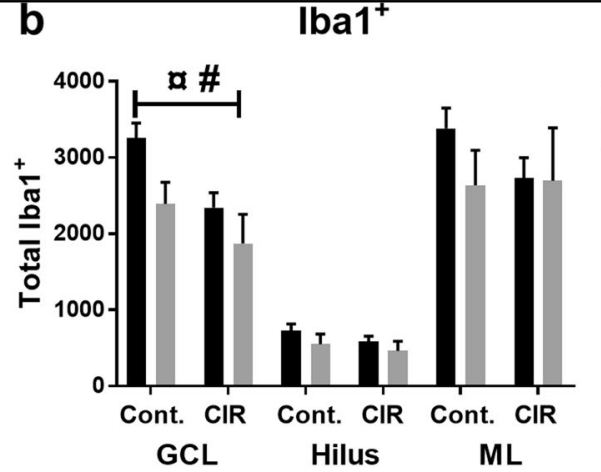

d

Olig2 $^{+}$

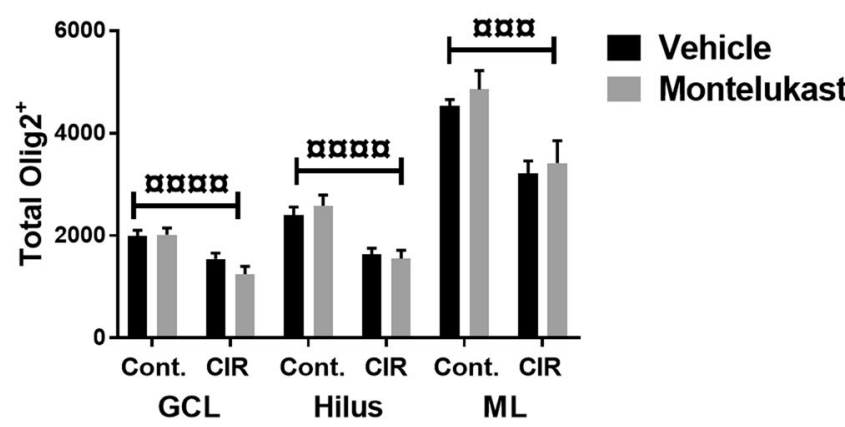

f

$\mathrm{S} 100^{+}$

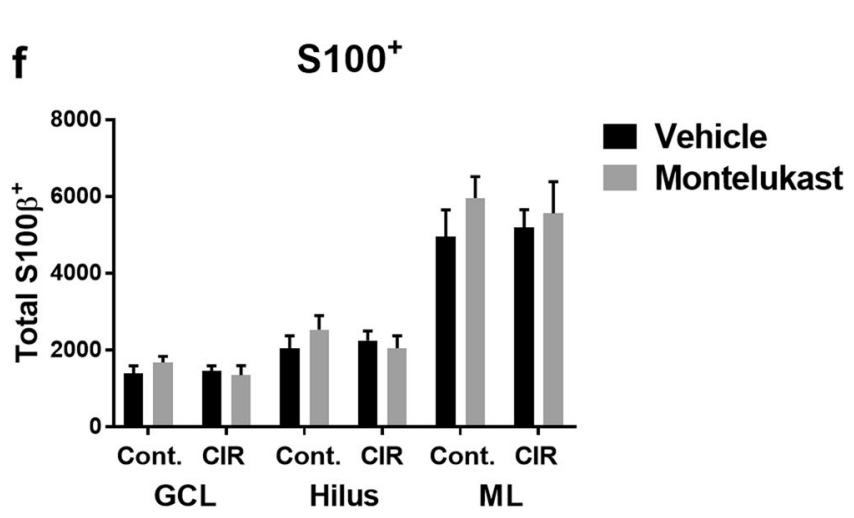

Montelukast
Vehicle

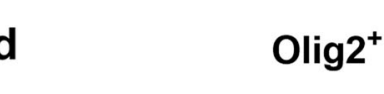

Fig. 5 Effects on non-neuronal cells in the dentate gyrus following CIR and montelukast treatment. A microphotograph (a) and quantifications $(\mathbf{b})$ of microglia $\left(\mathrm{Iba}^{+}\right)$in subregions of the dentate gyrus $(\mathrm{GCL}$, hilus, and $\mathrm{ML})$. A microphotograph $(\mathbf{c})$ of oligodendrocytes $\left(\mathrm{Olig} 2^{+}\right.$), and subsequent quantifications in subregions of the dentate gyrus (d). A microphotograph (e) and quantifications (f) of astrocytes (S100 $\left.{ }^{+}\right)$in subregions of the dentate gyrus. CIR cranial irradiation, GCL granule cell layer, Iba1 ionized calcium-binding adapter molecule 1, ML molecular layer, Olig2 oligodendrocyte transcription factor 2, S100 S100 calcium-binding protein. $\mathbf{a}, \mathbf{c}$, e Scale bar $=50 \mu \mathrm{m}$. $\mathbf{b} n_{\text {control vehicle }}=8, n_{\text {control montelukast }}=9$, $n_{\mathrm{CIR} \text { vehicle }}=6, n_{\mathrm{CIR} \text { montelukast }}=5 . \mathbf{d} n_{\text {control vehicle }}=7, n_{\text {control montelukast }}=8, n_{\mathrm{CIR} \text { vehicle }}=6, n_{\mathrm{CIR} \text { montelukast }}=5$. $\mathbf{f} n_{\text {control vehicle }}=10$, $n_{\text {control montelukast }}=9, n_{\text {CIR vehicle }}=6, n_{C I R}$ montelukast $=7$. Statistical results from two-way ANOVA are presented in the figures, $a=$ treatment

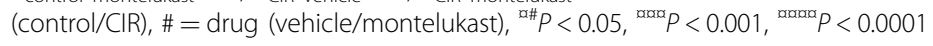

the same dose as in the aging study, $10 \mathrm{mg} / \mathrm{kg}$, but during a shorter time course compared to the clinical situation. Our aim was to investigate if this dose of montelukast could have similar protective effects after CIR, as the damage resembles the aging brain with elevated inflammation and less proliferation. Nevertheless, our findings could also contribute to explaining some alterations that montelukast induce in the young healthy brain as we have seen negative effects both acutely and after 2 weeks of daily administration of montelukast. However, more studies are needed to explore the therapeutic window for montelukast in pediatric patients. It should be emphasized that asthma itself can induce hypoxia in the brain, leading to, for example, cognitive dysfunction ${ }^{34}$. A future perspective could be to investigate if montelukast is a good treatment in such cases to control asthma and 
at the same time minimize injuries from the hypoxia for this group of children.

In summary, montelukast has negative effects on the maturation of the GCL during normal conditions, whereas during a pathological condition, such as following CIR, the effects can be protective. These findings, with the affected proliferation during normal conditions, in combination with the new profile for psychiatric adverse drug reactions, suggests that prescribing montelukast to young children should be a well thought through decision. However, more studies are needed to investigate if the negative effects are occurring also at lower dose spans and if the effect is chronic if ending the treatment with montelukast.

\section{Material and methods}

\section{Animals}

C57BL/6J mice with six female pups per mother were ordered from Charles River Laboratories, Germany. Animals were housed according to normal procedures at the Experimental Biomedicine animal facility (University of Gothenburg, Gothenburg, Sweden). The mice were kept on a 12-h light cycle with food and water provided ad libitum. The room temperature was $19-21^{\circ} \mathrm{C}$ with 40-70\% relative humidity. Animal experiments were approved by the Gothenburg committee of the Swedish Animal Welfare Agency (2015-72).

\section{Montelukast treatment}

Montelukast sodium powder (Sigma, USA) was dissolved in $99.5 \%$ ethanol, diluted 1:10 with $0.9 \%$ saline $(\mathrm{NaCl})$, and administered by intraperitoneal injections at a dose of $10 \mathrm{mg} / \mathrm{kg}$. Animals $(n=6-10)$ received daily injections of montelukast or vehicle $(0.9 \% \mathrm{NaCl}$ with $10 \%$ ethanol) starting 2 days before CIR, resulting in four doses for the acute study and 14 doses for the sub-acute study (Fig. 1a).

\section{Cranial irradiation procedure}

A linear accelerator True Beam STX (600 MU/min, 5.6 Gy/min; Radiation Oncology Systems, USA) with $6 \mathrm{MV}$ nominal photon energy was used for CIR as described earlier $^{28}$. Briefly, all animals were anesthetized on postnatal day 21 with a mixture of oxygen and isoflurane (Attane Vet, VM Pharma AB, Sweden) to immobilize the animals during the procedure. The whole brain was irradiated with a clinically relevant dose of $2 \times 4$ Gy with 12-h interval, using a radiation field of $2 \times 2 \mathrm{~cm}$, a source to skin distance of $99.5 \mathrm{~cm}$, and a dose variation of $\pm 5 \%$. After CIR, animals were returned to their dams. Control animals were anesthetized but did not receive CIR. Typically, pediatric patients with brain tumors are treated with radiotherapy, once a day, 5 days per week, for several weeks. The treatment protocol varies depending on tumor type and other relevant factors, but often it is $50-59.4 \mathrm{~Gy}$ in 28-33 fractions of $1.8 \mathrm{~Gy}$. This study was designed to investigate the radiation-induced injury in the normal tissue (8 Gy given in two fractions), hence a much lower dose than the tumor bed receive.

\section{Acute tissue preparation}

Brains were quickly removed after sacrifice, put in liquid nitrogen and stored at $-80^{\circ} \mathrm{C}$. Brains were homogenized by sonication in phosphate-buffered saline containing Triton X-100 (Merck KGaA, Germany), ethylenediaminetetraacetic acid (EDTA, Sigma-Aldrich, USA), and protease inhibitor cocktail (cOmplete, EDTAfree, Roche, Switzerland). Samples were then centrifuged and supernatant stored at $-80^{\circ} \mathrm{C}$. Protein concentration was measured using the Pierce BCA protein Assay Kit (Thermo Scientific, USA) according to the protocol provided by the manufacturer.

\section{Sub-acute tissue preparation}

Animals were anesthetized with sodium pentobarbital (Pentothal, Electra-box Pharma, Sweden) and transcardially perfused with $0.1 \mathrm{M}$ phosphate buffer $(\mathrm{pH} 7.5)$ to rinse the vascular system, followed by $6 \%$ formaldehyde (pH 7.4; Histofix; Histolab Products AB, Sweden). Brains were gently removed, immersion-fixed in Histofix for $24 \mathrm{~h}$ and stored in a sucrose solution (30\% sucrose in $0.1 \mathrm{M}$ phosphate buffer, $\mathrm{pH}$ 7.5). The right hemisphere was cut sagittally into $25 \mu \mathrm{m}$ sections in a series of 12, using a sliding microtome (SM2010R, Leica Microsystems, Germany), and stored at $4{ }^{\circ} \mathrm{C}$ in a cryoprotectant solution (25\% ethylene glycol and $25 \%$ glycerol).

\section{Blood collection}

Mice were anesthetized with a mixture of oxygen and isoflurane, blood was drawn from the heart with a $1 \mathrm{~mL}$ syringe (Omnifix ${ }^{\circledR}$, BRAUN, Germany) and centrifuged for $5 \mathrm{~min}$. Serum was collected and stored at $-80^{\circ} \mathrm{C}$.

\section{Fluorometric assay of caspase-3-like activity}

Caspase-dependent cell death was measured using a caspase activity assay. An aliquot of $20 \mu \mathrm{l}$ tissue homogenate was added to a microplate and mixed with $80 \mu \mathrm{l}$ extraction buffer ( $n=5$, duplicate samples) and analyzed as described earlier ${ }^{35}$. Cleavage of Ac-DEVD-AMC (for caspase-3/7-activity, Peptide Institute, Japan, cat. no.3171-v) was measured and expressed as pmol AMC released per mg protein and minute.

\section{Enzyme-linked immunosorbent assay}

Enzyme-linked immunosorbent assays (ELISA) were used to investigate chemokine $(\mathrm{C}-\mathrm{C}$ motif) ligand 2 (CCL2, MJE00, R\&D Systems, USA) and cluster of differentiation 68 (mouse CD68, EKM1518, Nordic BioSite, 
Sweden) expression. Analyses were performed according to instructions of the manufacturers and the amount of investigated proteins measured using a SpectraMax i3x (Molecular Devices, USA).

\section{Serum NFL}

Serum sample neurofilament light chain (NFL) concentration was determined using an in-house NFL assay on the single molecule array (Simoa) platform, which has been described in detail previously ${ }^{36}$. Briefly, paramagnetic carboxylated beads (Quanterix Corp, USA) were coated with a mouse anti-NFL antibody (UD1, UmanDiagnostics, Sweden) and incubated with sample and a biotinylated mouse anti-NFL antibody (UD2, UmanDiagnostics, Sweden) in a Simoa HD-1 instrument (Quanterix).

\section{Immunohistochemistry}

Sections were treated as follows: rinsed (always in Trisbuffered saline [TBS], $50 \mathrm{mM}$ Tris- $\mathrm{HCl}$ in $150 \mathrm{mM} \mathrm{NaCl}$ ), incubated in $0.6 \% \mathrm{H}_{2} \mathrm{O}_{2}$ in TBS for $30 \mathrm{~min}$, rinsed and incubated for $30 \mathrm{~min}$ in a TBS block solution with $3 \%$ donkey serum (Jackson ImmunoResearch Laboratories Inc, USA), and $0.1 \%$ Triton X-100 (Merck KGaA, Germany). Sections were incubated overnight at $4{ }^{\circ} \mathrm{C}$ in block solution using the following primary antibodies: oligodendrocyte transcription factor 2 (1:500, rabbit anti-Olig2, ab109186, Abcam, UK), marker of proliferation Ki-67 (1:1000, rabbit anti-Ki-67, ab15580, Abcam, UK), S100 calcium-binding protein (1:2000, mouse anti-S100, MA512969, ThermoFisher Scientific, USA), rabbit anti-ionized calcium-binding adapter molecule 1 (1:1000, rabbit antiIba1, 019-19741, WAKO Pure Chemical Industries ltd, Japan), and doublecortin (1:500, polyclonal goat antiDCX, Santa Cruz Biotechnology, USA). Sections were rinsed, incubated for $1 \mathrm{~h}$ with block solution and biotinylated secondary antibodies (1:1000, Jackson ImmunoResearch Laboratories Inc, USA), rinsed and incubated with avidin-biotin-peroxidase $(10 \mu \mathrm{L} / \mathrm{mL}$ TBS of $\mathrm{A}$ and $\mathrm{B}$, Vectastain Elite ABC Kit, Vector Laboratories, USA) for $1 \mathrm{~h}$. Sections were rinsed and developed in 3,3'-diaminobenzidine (DAB, Saveen Werner AB, Sweden) diluted in TBS with $\mathrm{H}_{2} \mathrm{O}_{2}$ and $\mathrm{NiCl}_{2}$, until sufficient color was noted. After rinsing in tap water, sections were mounted using $0.1 \mathrm{M}$ phosphate buffer, $\mathrm{pH} 7.5$, and dried overnight, then cover slipped with X-Tra-Kitt (Medite GmbH, Germany).

\section{NeuroTrace Fluorescent Nissl stain}

Sections were rinsed in TBS and then washed in TBS with $0.1 \%$ Triton X-100 for 10 min. After further washing, sections were incubated with NeuroTrace ${ }^{\mathrm{TM}}$ 500/525 Green Fluorescent Nissl stain for $20 \mathrm{~min}$ (N21480, ThermoFisher Scientific, USA). Following several rinsing steps in TBS, the sections were mounted and cover slipped with ProLong ${ }^{\circledR}$ Gold Antifade Reagent (ThermoFisher Scientific, USA).

\section{Stereological procedures}

Cells were counted in every 12th section using systematic-random sampling (Stereoinvestigator, MicroBrightField, USA) and a Leica DM6000 B microscope (Leica Microsystems, Germany). Counting started on sections containing a clearly divided dorsal and ventral hippocampus (only the dorsal granule cell layer [GCL] was measured). Total volumes were calculated according to the Cavalieri principle $(V=\mathrm{SA} \times P \times T$, where $V$ is the total volume, SA is the sum of area measurements, $P$ is the inverse of the sampling fraction, and $T$ is the section thickness). The total number of cells was obtained by dividing the number of counted cells with the sampling fraction. Volumes for corpus callosum and thalamus were also measured in sections eligible for hippocampal quantifications.

NeuroTrace was used to quantify neurons in GCL $(\times 40$ objective). A $275 \times 75-\mu \mathrm{m}$ grid was randomly placed over the traced area and counting frames $(25 \times 25 \mu \mathrm{m})$ were placed within the grid. The total number of GCL neurons per animal was calculated by dividing the number of counted cells with the sampling fractions, i.e., fraction of sampling area/total traced area $\times$ series fraction $(1 / 12) \times$ optical dissector height/physical section thickness.

\section{Statistics}

For statistical analyses, two-way ANOVA was used (drug and treatment as main effects), followed by a post hoc test (Sidak, corrected for multiple testing using GraphPad Prism 7.02). Weight was analyzed using threeway ANOVA (Stata). Statistical significance was considered if $P<0.05$.

\section{Acknowledgements}

We are grateful for the skillful technical assistance of Rita Grandér and thank our former and current students, Jolie Danial, Alba Rodríguez-Rodríguez, and Zainab Jasim. This study was supported by grants from the Swedish and European Research Councils, the Knut and Alice Wallenberg Foundation, the Swedish Brain Foundation, Swedish State Support for Clinical Research, the Wolfson Foundation, Alzheimerfonden, the Swedish Society of Medicine, the Swedish Childhood Cancer Foundation (Barncancerfonden), the Frimurare Barnhus Foundations of Gothenburg, the Lions Cancer Foundation West, the Wilhelm and Martina Lundgren Foundation, the Edith Jacobssons Donation Fund, the Torsten Söderberg Foundation, and Jubileumskliniken's anti-cancer research fund.

\section{Author details}

${ }^{1}$ Department of Pharmacology, Institute of Neuroscience and Physiology, Sahlgrenska Academy, University of Gothenburg, Gothenburg, Sweden. ${ }^{2}$ Department of Oncology, Institute of Clinical Sciences, Sahlgrenska Academy, University of Gothenburg, Gothenburg, Sweden. ${ }^{3}$ Department of Psychiatry and Neurochemistry, Institute of Neuroscience and Physiology, Sahlgrenska Academy, University of Gothenburg, Mölndal, Sweden. ${ }^{4}$ Clinical

Neurochemistry Laboratory, Sahlgrenska University Hospital, Gothenburg, Sweden. ${ }^{5}$ Department of Molecular Neuroscience, UCL Institute of Neurology, 
Queen Square, London, UK. 'UK Dementia Research Institute, UCL, London, UK. ${ }^{7}$ Center for Brain Repair and Rehabilitation, Institute of Neuroscience and Physiology, Sahlgrenska Academy, University of Gothenburg, Gothenburg Sweden. ${ }^{8}$ Center for Stroke Research Berlin, Charité - Universitätsmedizin, Berlin, Germany

\section{Conflict of interest}

Authors K.B. and H.Z. are co-founders of Brain Biomarker Solutions in Gothenburg AB, a GU Ventures-based platform company at the University of Gothenburg. H.Z. has served at advisory boards of Eli Lilly and Roche Diagnostics and has received travel support from Teva. The remaining authors declare that they have no conflict of interest.

\section{Publisher's note}

Springer Nature remains neutral with regard to jurisdictional claims in published maps and institutional affiliations.

Received: 16 March 2018 Revised: 8 June 2018 Accepted: 11 June 2018 Published online: 10 July 2018

\section{References}

1. Global Initiative for Asthma. Global Strategy for Asthma Management and Prevention www.ginasthma.org (2016).

2. Bisgaard, $\mathrm{H}$. et al. Safety and tolerability of montelukast in placebo-controlled pediatric studies and their open-label extensions. Pediatr. Pulmonol. 44 568-579 (2009).

3. Benard, B. et al. Neuropsychiatric adverse drug reactions in children initiated on montelukast in real-life practice. Eur. Respir. J. 50, 700148 (2017).

4. Bygdell, M., Brunlof, G., Wallerstedt, S. M. \& Kindblom, J. M. Psychiatric adverse drug reactions reported during a 10-year period in the Swedish pediatric population. Pharmacoepidemiol. Drug Saf. 21, 79-86 (2012).

5. Wallerstedt, S. M., Brunlof, G., Sundstrom, A. \& Eriksson, A. L. Montelukast and psychiatric disorders in children. Pharmacoepidemiol. Drug Saf. 18, 858-864 (2009).

6. Phillis, J. W., Horrocks, L. A. \& Farooqui, A. A. Cyclooxygenases, lipoxygenases, and epoxygenases in CNS: their role and involvement in neurological disorders. Brain Res. Rev. 52, 201-243 (2006).

7. Marschallinger, J. et al. Structural and functional rejuvenation of the aged brain by an approved anti-asthmatic drug. Nat. Commun. 6, 8466 (2015).

8. Zhao, R., Shi, W. Z., Zhang, Y. M., Fang, S. H. \& Wei, E. Q. Montelukast, a cysteinyl leukotriene receptor-1 antagonist, attenuates chronic brain injury after focal cerebral ischaemia in mice and rats. J. Pharm. Pharmacol. 63, 550-557 (2011).

9. Segal, D. \& Karajannis, M. A. Pediatric brain tumors: an update. Curr. Probl. Pediatr. Adolesc. Health Care 46, 242-250 (2016).

10. Zapotocky, M., Ramaswamy, V., Lassaletta, A. \& Bouffet, E. Adolescents and young adults with brain tumors in the context of molecular advances in neuro-oncology. Pediatr. Blood Cancer 65, (2018) https://doi.org/10.1002/ pbc.26861.

11. Han, J. W. et al. Comprehensive clinical follow-up of late effects in childhood cancer survivors shows the need for early and well-timed intervention. Ann. Oncol. 20, 1170-1177 (2009).

12. Meyers, C. A. \& Brown, P. D. Role and relevance of neurocognitive assessment in clinical trials of patients with CNS tumors. J. Clin. Oncol. 24, 1305-1309 (2006).

13. Belka, C., Budach, W., Kortmann, R. D. \& Bamberg, M. Radiation induced CNS toxicity-molecular and cellular mechanisms. Br. J. Cancer 85, 1233-1239 (2001).
14. Kalm, M. et al. Transient inflammation in neurogenic regions after irradiation of the developing brain. Radiat. Res. 171, 66-76 (2009).

15. Kalm, M., Lannering, B., Bjork-Eriksson, T. \& Blomgren, K. Irradiation-induced loss of microglia in the young brain. J. Neuroimmunol. 206, 70-75 (2009).

16. Fike, J. R., Rosi, S. \& Limoli, C. L. Neural precursor cells and central nervous system radiation sensitivity. Semin. Radiat. Oncol. 19, 122-132 (2009).

17. Kalm, M., Karlsson, N., Nilsson, M. K. \& Blomgren, K. Loss of hippocampal neurogenesis, increased novelty-induced activity, decreased home cage activity, and impaired reversal learning one year after irradiation of the young mouse brain. Exp. Neurol. 247, 402-409 (2013).

18. Makale, M. T., McDonald, C. R., Hattangadi-Gluth, J. A. \& Kesari, S. Mechanisms of radiotherapy-associated cognitive disability in patients with brain tumours. Nat. Rev. Neurol. 13, 52-64 (2017).

19. Jenrow, K. A. et al. Selective inhibition of microglia-mediated neuroinflammation mitigates radiation-induced cognitive impairment. Radiat. Res. 179, 549-556 (2013).

20. Monje, M. L., Toda, H. \& Palmer, T. D. Inflammatory blockade restores adult hippocampal neurogenesis. Science 302, 1760-1765 (2003).

21. Belarbi, K., Jopson, T., Arellano, C., Fike, J. R. \& Rosi, S. CCR2 deficiency prevents neuronal dysfunction and cognitive impairments induced by cranial irradiation. Cancer Res. 73, 1201-1210 (2013).

22. Blomstrand, M., Kalm, M., Grander, R., Bjork-Eriksson, T. \& Blomgren, K. Different reactions to irradiation in the juvenile and adult hippocampus. Int. J. Radiat. Biol. 90, 807-815 (2014).

23. Kalm, M. et al. C3 deficiency ameliorates the negative effects of irradiation of the young brain on hippocampal development and learning. Oncotarget 7, 19382-19394 (2016)

24. Zhang, L. et al. Minocycline ameliorates cognitive impairment induced by whole-brain irradiation: an animal study. Radiat. Oncol. 9, 281 (2014).

25. Barlind, A. et al. The growth hormone secretagogue hexarelin increases cell proliferation in neurogenic regions of the mouse hippocampus. Growth Horm. IGF Res. 20, 49-54 (2010).

26. Roughton, K., Bostrom, M., Kalm, M. \& Blomgren, K. Irradiation to the young mouse brain impaired white matter growth more in females than in males. Cell Death Dis. 4, e897 (2013).

27. Fukuda, A. et al. Age-dependent sensitivity of the developing brain to irradiation is correlated with the number and vulnerability of progenitor cells. J. Neurochem. 92, 569-584 (2005)

28. Kalm, M., et al. Serum concentrations of the axonal injury marker neurofilament light protein are not influenced by blood-brain barrier permeability. Brain Res. 1668,12-19 (2017).

29. Huber, C. et al. Inhibition of leukotriene receptors boosts neural progenitor proliferation. Cell. Physiol. Biochem. 28, 793-804 (2011).

30. Ballerini, P. et al. P2Y1 and cysteinyl leukotriene receptors mediate purine and cysteinyl leukotriene co-release in primary cultures of rat microglia. Int. J. Immunopathol. Pharmacol. 18, 255-268 (2005).

31. Lecca, D. et al. The recently identified P2Y-like receptor GPR17 is a sensor of brain damage and a new target for brain repair. PLOS ONE 3, e3579 (2008).

32. Bordt, E. A. \& Polster, B. M. NADPH oxidase- and mitochondria-derived reactive oxygen species in proinflammatory microglial activation: a bipartisan affair? Free Radic. Biol. Med. 76, 34-46 (2014)

33. Liu, J. L., Zhao, X. H., Zhang, D. L., Zhang, J. B. \& Liu, Z. H. Effect of montelukast on the expression of interleukin-18, telomerase reverse transcriptase, and $\mathrm{BCl}-2$ in the brain tissue of neonatal rats with hypoxic-ischemic brain damage. Genet. Mol. Res. 14, 8901-8908 (2015).

34. Guo, R. B. et al. Chronic asthma results in cognitive dysfunction in immature mice. Exp. Neurol. 247, 209-217 (2013).

35. Wang, $X$. et al. Caspase-3 activation after neonatal rat cerebral hypoxiaischemia. Biol. Neonate 79, 172-179 (2001).

36. Kuhle, J. et al. Comparison of three analytical platforms for quantification of the neurofilament light chain in blood samples: ELISA, electrochemiluminescence immunoassay and Simoa. Clin. Chem. Lab. Med. 54 1655-1661 (2016) 